\title{
Antibiotic susceptibility and resistance profiles of Romanian Clostridioides difficile isolates
}

\author{
Ioana S. Macovei ${ }^{1 *}$, Daniela Lemeni ${ }^{1}$, Roxana Șerban ${ }^{2}$, Andreea Niculcea ${ }^{2}$, \\ Gabriel A. Popescu ${ }^{3}$, Maria Nica $^{4}$, Anca Petrini $^{5}$, Grigore Mihăescu ${ }^{6}$ \\ 1. "Cantacuzino" National Medico-Military Institute for Research and \\ Development, Bucharest, Romania \\ 2. National Institute for Public Health Bucharest \\ 3. "Matei Balş, National Institute for Infectious Diseases \\ 4. "Victor Babeș" Infectious and Tropical Disease Hospital \\ 5. "Carol Davila" Nephrology Hospital \\ 6. Faculty of Biology, University of Bucharest
}

\begin{abstract}
This study investigated the antibiotic susceptibility patterns and genetic resistance markers of 35 C. difficile strains isolated from patients with C. difficile infection. Vancomycin, metronidazole, tigecycline, teicoplanin, rifampicin, moxifloxacin, cefotaxime, tetracycline, erythromycin, clindamycin, chloramphenicol, linezolid and imipenem MICs were determined for toxigenic strains belonging to PCR ribotypes (PR) 012 (2), 014 (4), 017 (3), 018 (2), 027 (17), 046 (2), 087 (3) and 115 (2). Results showed vancomycin, metronidazole, tigecycline and teicoplanin to be active against all isolates. High resistance rates were noticed against cefotaxime $(n=35)$, clindamycin $(n=$ $33)$, imipenem $(n=31)$, moxifloxacin $(n=25)$, erythromycin $(n=25)$ and rifampicin $(n=22)$. Linezolid-resistance was found in three isolates (PR 017/2, PR 012/1), showing complex resistance (7-9 antibiotics). PR 012, 017, 018, 027 and 046 isolates $(n=26)$ were resistant to 5-9 antibiotics. Twelve resistance profiles (2-9 antibiotics) were detected. Rifampicin-moxifloxacin-cefotaxime-erythromycin-clindamycin-imipenem-resistance was predominant, being expressed by 18 strains (PR 027/17, PR 018/1). PCR results suggested tetracycline-resistance to be induced by the gene tetM. Three tetM-positive isolates (PRs 012, 046), were also tndX-positive, suggesting the presence of a Tn5397-like element. Only two MLSB-resistant strains (PR 012) had the ermB gene and chloramphenicol-resistance determinant catD was not detected, leaving room for further investigating resistance mechanisms. Multidrug resistance could be attributed to most analysed strains, underlining, once more, the impact of wide-spectrum antimicrobial over prescription, still a tendency in our country, on transmission of antimicrobial resistance and emergence of epidemic $C$. difficile strains generating outbreaks.
\end{abstract}

Keywords: C. difficile, antibiotic susceptibility, resistance profile PCR ribotype

Received: $21^{\text {st }}$ August 2017; Accepted: $10^{\text {th }}$ January 2018; Published: $5^{\text {th }}$ February 2018

*Corresponding author: Ioana Macovei, "Cantacuzino" National Medico-Military Institute for Research and Development, Bucharest, Romania. E-mail: ioanas.macovei@gmail.com 


\section{Introduction}

Clostridioides difficile is an anaerobic, Gram positive spore-forming bacillus, considered the etiologic agent of hospital - or community-acquired post-antibiotic diarrhoea and pseudomembranous colitis (PMC) [1]. The bacterium is ubiquitous in the environment: spores can be found in soil, lake waters or sediments, but may also colonize the large intestine of pets (dogs, cats) or farm animals [2, 3]. It was first discovered in the gut microbiota of healthy newborns in 1935 [4]. Most C. difficile strains are toxin-producing, but there are also non-toxigenic, clinically irrelevant strains. Toxin A (TcdA enterotoxin) and toxin B (TcdB - cytotoxin) are the main virulence factors, although important epidemic strains also produce a third type - the binary toxin $(C$. difficile toxin - CDT) [5]. The main risk factors for colonization with toxigenic $C$. difficile and associated disease are considered to be hospitalisation in acute or long-term care units, advanced age ( $\geq 65$ years) $[1,6]$ and prolonged treatment with wide-spectrum antibiotics like clindamycin, cephalosporins or fluoroquinolones, to which many $C$. difficile strains are non-susceptible [2, 6-10]. C. difficile infection (CDI) may also be community-associated, targeting both paediatric ( $>2$ years) and adult patients $[2,11]$, through contact with spore-contaminated surfaces, infected persons, or as zoonotic infections [3]. Many clinical isolates in Europe, including those belonging to PCR ribotypes (PR) 001, 012, 017, 018, 027 or 078, are considered multi-drug resistant (MDR) $[12,13]$. Resistance patterns often target antibiotics from the Macrolide-Lincosamide-Streptogramin B $\left(\mathrm{MLS}_{\mathrm{B}}\right)$ family, tetracycline, chloramphenicol, rifaximin and fluoroquinolones, especially in hospital settings with high rates of clindamycin, rifampin or fluoroquinolone administration. Clindamycin administration was historically defined as a major risk factor for CDI [7]. Presently, it is sug- gested to represent a lower risk of infection with the major epidemic type PR 027 emerged in the last decades, compared to fluoroquinolones [10, 14]. However, high levels of $\mathrm{MLS}_{\mathrm{B}}$-resistance are still being reported for other epidemic PRs, such as 001,017 or 078 [12]. Although MICs may vary, $C$. difficile strains are considered constitutively resistant to cephalosporins, through mechanisms that are insufficiently known at this moment [10].

Antibiotic resistance is frequently transmitted between bacterial strains through mobile genetic elements. The best described genetic determinant was considered class B erythromycin ribosomal methylase gene (ermB), harboured by Tn5398, Tn5398-like or other transposons [10, $16,17]$. Resistance to tetracycline is induced, in most cases, by a ribosome protection protein (RPP) - TetM [18] - encoded by the tet $\mathrm{M}$ gene, carried by $\operatorname{Tn} 5397$ or other Tn916-like transposons [10, 19]. Tn5397, first identified in $C$. difficile strains [19], also carries the gene tndX, encoding a protein responsible for the insertion and excision of the element [20]. tndX is used as a marker for the detection of Tn5397 in C. difficile strains [21]. In C. difficile, tetracycline-resistance may also be mediated by gene tet $\mathrm{W}$, sometimes co-present with tet $\mathrm{M}[22,23]$. Chloramphencol-resistance may be induced by chloramphenicol acetyltransferase, encoded by the catD gene, specific to $\operatorname{Tn} 4453 a$ or $\operatorname{Tn} 4453 b$ transposons [10, 17]. CDI has represented a considerable burden for public health in North America, Asia and Europe in the last decades $[6,24]$.

In Romania, high rates persisted since first outbreaks occurred in 2011, with PR 027 as prevalent among the strain types causing outbreaks [25-27]. One of the main causes for the persistence of high CDI rates may be the over prescription of wide spectrum antibiotics, still an issue in our country. However, antimicrobial susceptibility or resistance patterns of $C$. diffi- 
cile strains involved in Romanian infections are insufficiently described at this moment. For this purpose, the antibiotic susceptibility of $C$. difficile strains, isolated from Romanian patients with antibiotic-associated diarrhoea, was analysed in the present study. Molecular mechanisms of resistance were also investigated.

\section{Materials and Methods}

\section{In vitro antibiotic susceptibility testing}

The study was based on the analysis of 35 non-duplicate toxigenic $C$. difficile strains isolated between 2013 and 2017 from patients with antibiotic-associated diarrhoea confirmed as $C$. difficile infection by rapid immunochromatographic toxin A/B screening tests and toxigenic culture. PRs of the isolates were identified in previous studies $[25,28]$. Seventeen strains belonging to PR 027 were included. The rest of the analysed isolates belonged to 7 other PRs, as follows: $017(\mathrm{n}=3), 087(\mathrm{n}=3), 014(\mathrm{n}=4)$, $046(\mathrm{n}=2), 018(\mathrm{n}=2), 012(\mathrm{n}=2)$ and $115(\mathrm{n}$ $=2$ ). Antibiotic susceptibility profiles and MICs were determined using Etest (bioMérieux, France), according to manufacturer's instructions. Susceptibility to the following antibiotics was tested: vancomycin (VAN), metronidazole (MTR), tigecycline (TGC), teicoplanin (TEC), rifampicin (RIF), moxifloxacin (MFX), cefotaxime (CTX), tetracycline (TCY), erythromycin (ERY), clindamycin (CLI), chloramphenicol (CHL), linezolid (LNZ) and imipenem (IPM). Bacterial suspensions of 1.0 McFarland density, prepared from 24-48h cultures, were inoculated on Brucella blood agar plates (BBA Agar, bioMérieux, France). After $48 \mathrm{~h}$ plate incubation in anaerobiosis, MIC values were recorded and interpreted using EUCAST breakpoints or epidemiological cut-off (ECOFF) MIC values for C. difficile, CLSI breakpoints for Gram positive anaerobes and literature references as guidance [9, 29-31].

\section{PCR detection of genetic determinants of antibiotic resistance}

The presence of genes known to mediate resistance to TCY, $\mathrm{MLS}_{\mathrm{B}}$ family and CHL was also analysed [10]. DNA samples were extracted from $C$. difficile colonies grown on Columbia blood agar (COS Agar plates, bioMérieux, France), using a resin extraction kit (InstaGene Matrix, Bio-Rad, USA), according to the manufacturer's instructions. The presence of antibiotic resistance genes tet $\mathrm{M}$, erm $\mathrm{B}$ and cat $\mathrm{D}$ was simultaneously tested, using a multiplex PCR protocol, as previously described [17]. The reaction mix contained specific pairs of primers $\left(5^{\prime} \rightarrow 3\right.$ '): TETMd (TGGAATTGATTTATCAACGG) and TETMr (TTCCAACCATACAATCCTTG) for tet $\mathrm{M}$, [31], E5 (CTCAAAACTTTTTAACGAGTG) and E6 (CCTCCCGTTAAATAATAGATA) for ermB and CL1 (ATACAGCATGACCGTTAAAG) / CL2 (ATGTGAAATCCGTCACATAC) for cat $\mathrm{D}$ [17]. The PCR reaction was performed with $0,3-0,6 \mathrm{pmol} / \mu 1$ of primers, a ready-to-use mix containing Taq polymerase (Qiagen Multiplex PCR, USA) and $5 \mu$ of DNA sample, in a final volume of $50 \mu 1$. The amplification parameters were set according to the above mentioned protocol [17]. Simplex PCR was performed for the detection of the tet $\mathrm{W}$ gene, using the WRC1/ WRC2 pair of primers previously published [23]. Another PCR protocol was used to determine the presence of gene $t n d X$, a Tn5367-like marker, with the specific set of primers TNDX1 and TNDX2, described by Spigaglia et al. [21]. The PCR products were verified with conventional gel electrophoresis, using 1.5\% agarose gels stained with ethidium bromide. The expected amplification products were fragments of: $1080 \mathrm{bp}$ (tet $\mathrm{M}), 711 \mathrm{bp}($ ermB) and $500 \mathrm{bp}$ $($ cat $\mathrm{D})$ in the multiplex PCR, $457 \mathrm{bp}($ tet $\mathrm{W})$ and $1600 \mathrm{bp}($ tndX) for the other PCR protocols. 


\section{Results}

\section{In vitro antibiotic susceptibility}

Results showed VAN, MTR and TGC to be highly active against the analysed group of isolates: geometric mean (GM) MIC was 0.415 $\mu \mathrm{g} / \mathrm{ml}$ for VAN, $0.11 \mu \mathrm{g} / \mathrm{ml}$ for MTR and 0.021 $\mu \mathrm{g} / \mathrm{ml}$ for TCG, as presented in Table 1 . The highest MTR MIC values $-0.75 \mu \mathrm{g} / \mathrm{ml}$ and 1 $\mu \mathrm{g} / \mathrm{ml}$ - were found in strains belonging to PR 027, among the analysed PRs [data not shown]. TEC susceptibility breakpoints or ECOFF values were not available for $C$. difficile or Gram positive anaerobes [29]. However, the low MICs resulted $(\mathrm{GM} \mathrm{MIC}=0.082 \mu \mathrm{g} / \mathrm{ml})$ suggest the glycopeptide to be active against the analysed $C$. difficile strains (Table 1, Figure 1)
Antibiotic resistance was noticed especially in isolates belonging to PRs 012, 017, 018, 027 and $046(\mathrm{n}=26)$, which were non-susceptible to 5-9 antibiotics, while PRs 014, 087 and 115 ( $\mathrm{n}=$ 9) showed less complex resistance profiles (2-3 antibiotics). None of the isolates was susceptible to CTX (Figure 1). MICs ranged from 64 to 256 $\mu \mathrm{g} / \mathrm{ml}$, most strains being highly resistant (Table 2). The analysed group of isolates consisted of both susceptible and non-susceptible strains to the remaining antibiotics (RIF, MFX, TCY, ERY, CLI, CHL, LNZ and IPM). Susceptibility and MIC values are summarised in Table 1.

A high resistance rate, represented by strains from all the ribotypes included in the study, resulted against CLI (33/35), followed by resist-

Table 1. Susceptibility (MIC values) of the analysed group of $C$. difficile isolates $(n=35)$ to 13 antibiotics.

\begin{tabular}{|c|c|c|c|c|c|c|}
\hline Antibiotic & $\begin{array}{l}\text { Breakpoint } \\
(\mathrm{S})^{\mathrm{a}, \mathrm{b}}(\mu \mathrm{g} / \mathrm{ml})\end{array}$ & $\begin{array}{l}\text { Susceptible } \\
\text { / Resistant } \\
\text { isolates (n) }\end{array}$ & $\begin{array}{c}\text { Geometric } \\
\text { mean MICs } \\
(\mu \mathrm{g} / \mathrm{ml})\end{array}$ & $\begin{array}{c}\mathrm{MIC}_{50} \\
(\mu \mathrm{g} / \mathrm{ml})\end{array}$ & $\begin{array}{l}\mathrm{MIC}_{90} \\
(\mu \mathrm{g} / \mathrm{ml})\end{array}$ & MIC range \\
\hline VAN & $\leq 2$ & $35 / 0$ & 0.415 & 0.38 & 0.75 & $0.125-1$ \\
\hline MTR & $\leq 2$ & $35 / 0$ & 0.11 & 0.094 & 0.75 & $0.016-1$ \\
\hline TGC & $\leq 0.25$ & $35 / 0$ & 0.021 & 0.023 & 0.032 & $<0.016-0.047$ \\
\hline TEC $^{c}$ & - & - & 0.082 & 0.064 & 0.25 & $0.016-0.75$ \\
\hline RIF & $\leq 0.004$ & $13 / 22$ & 1.358 & $>32$ & $>32$ & $<0.002->32$ \\
\hline MFX & $\leq 4$ & $10 / 25$ & 17.139 & $>32$ & $>32$ & $0.38->32$ \\
\hline CTX & $\leq 16$ & $0 / 35$ & $>256$ & $>256$ & $>256$ & $64->256$ \\
\hline TCY & $\leq 0.25$ & $27 / 8$ & 0.21 & 0.064 & 12 & $0.016-32$ \\
\hline ERY & $\leq 2$ & $10 / 25$ & 85.721 & $>256$ & $>256$ & $0.25->256$ \\
\hline CLI & $\leq 2$ & $2 / 33^{d}$ & 11.835 & 6 & $>256$ & $1->256$ \\
\hline $\mathrm{CHL}$ & $\leq 8$ & $31 / 4^{\mathrm{e}}$ & 5.858 & 4 & 16 & $1->256$ \\
\hline LNZ & $\leq 4$ & $32 / 3$ & 2.162 & 2 & 4 & $0.5-32$ \\
\hline IPM & $\leq 4$ & $4 / 31^{\mathrm{f}}$ & 25.072 & $>32$ & $>32$ & $1.5->32$ \\
\hline
\end{tabular}

Abbreviations: VAN - vancomycin, MTR - metronidazole, TGC - tigecycline TEC - teicoplanin, RIF - rifampicin, MFX - moxifloxacin, CTX - cefotaxime, TCY - tetracycline, ERY - erythromycin, CLI - clindamicin, CHL - chloramphenicol, LNZ - linezolid, IPM - imipenem; $\mathrm{S}$ - susceptible, $\mathrm{R}$ - resistant, I - intermediary.

a) Breakpoints define susceptible isolates.

b) VAN, MTR - EUCAST clinical breakpoints for $C$. difficile; TGC, RIF, MFX, ERY - EUCAST epidemiological cut-off values (ECOFF) for $C$. difficile [42]; CTX, CLI, CHL, IPM - CLSI breakpoints for susceptible Gram positive anaerobes [41]; LNZ - published sources $[2,21]$.

c) Breakpoints or ECOFF MICs are not given for TEC. However, the antibiotic could be considered active against the strains, as the low MICs obtained might suggest.

d) $R=\sum_{R(n=26), I(n=7)^{\prime}}$;

e) $\quad \mathrm{R}=\sum_{\mathrm{R}(\mathrm{n}=3), \mathrm{I}(\mathrm{n}=1)^{\circ}}$.

f) $\quad R=\sum_{R(n=26), I(n=5)}^{R}$; 


\section{Resistant}

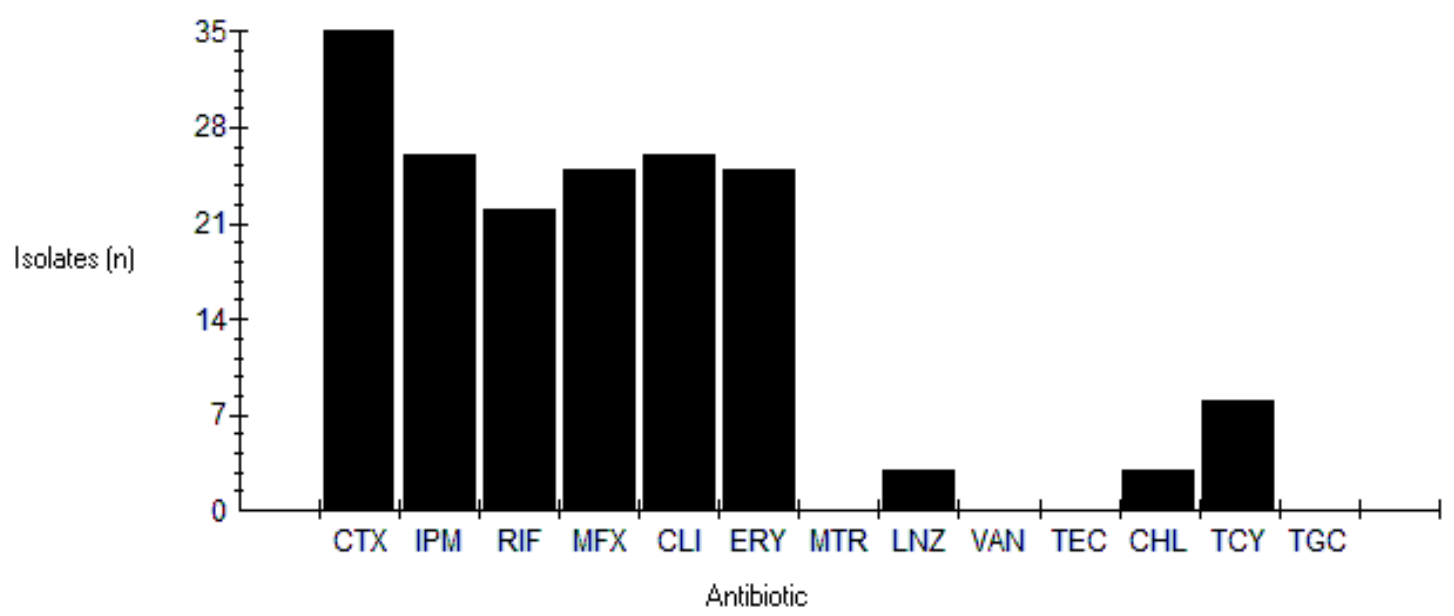

Figure 1. Antibiotic resistance rates of the analysed $C$. difficile isolates to the antibiotics tested: CTX - cefotaxime, IPM - imipenem, RIF - rifampicin, MFX - moxifloxacin, CLI - clindamycin, ERY erythromycin, MTR - metronidazole, LNZ - linezolid, VAN - vancomycin, TEC - teicoplanin, CHL chloramphenicol, TCY - tetracycline, TGC - tigecycline.

Table 2. Geometric mean MIC values resulted for PCR ribotypes

\begin{tabular}{|c|c|c|c|c|c|c|c|c|c|c|c|c|c|}
\hline \multirow{2}{*}{$\begin{array}{c}\text { PCR } \\
\text { Ribotype (n) }\end{array}$} & \multicolumn{13}{|c|}{ Geometric mean MICs $(\mu \mathrm{g} / \mathrm{ml})$} \\
\hline & VAN & MTR & TGC & TEC & RIF & MFX & CTX & TCY & ERY & CLI & CHL & LNZ & IPM \\
\hline $012(2)$ & 0.38 & 0.089 & 0.033 & 0.089 & 0.002 & 1.732 & $>256$ & 11.314 & $>256$ & $>256$ & 8 & 8 & $>32$ \\
\hline $014(4)$ & 0.555 & 0.078 & 0.019 & 0.121 & $<0.002$ & 0.892 & $>256$ & 0.05 & 1.456 & 6.447 & 2.913 & 1.316 & 19.596 \\
\hline $017(3)$ & 0.416 & 0.08 & 0.029 & 0.198 & $>32$ & $>32$ & $>256$ & 15.119 & 58.148 & 92.304 & 80.635 & 8.32 & $>32$ \\
\hline $018(2)$ & 0.612 & 0.078 & 0.027 & 0.75 & $>32$ & $>32$ & $>256$ & 0.375 & $>256$ & 6.928 & 3.464 & 2.449 & 11.314 \\
\hline 027 (17) & 0.361 & 0.137 & 0.018 & 0.047 & $>32$ & $>32$ & $>256$ & 0.072 & $>256$ & 6.14 & 4.476 & 1.747 & 30.935 \\
\hline 046 (2) & 0.534 & 0.063 & 0.027 & 0.108 & 0.002 & 0.38 & $>256$ & 13.856 & $>256$ & $>256$ & 11.314 & 1.732 & $>32$ \\
\hline 087 (3) & 0.33 & 0.115 & 0.02 & 0.047 & 0.002 & 20.966 & 184.608 & 0.059 & 0.63 & 2.08 & 4.579 & 1.5 & 6.604 \\
\hline $115(2)$ & 0.612 & 0.154 & 0.023 & 0.19 & $<0.002$ & 1.061 & 181.019 & 0.055 & 1.732 & 6 & 4.243 & 2.449 & 6.928 \\
\hline
\end{tabular}

Abbreviations: VAN - vancomycin, MTR - metronidazole, TGC - tigecycline, TEC - teicoplanin, RIF - rifampicin, MFX - moxifloxacin, CTX - cefotaxime, TCY - tetracycline, ERY - erythromycin, CLI - clindamicin, CHL - chloramphenicol, LNZ - linezolid, IPM - imipenem.

ance to $\operatorname{IPM}(31 / 35), \operatorname{MFX}(25 / 35), \operatorname{ERY}(25 / 35)$ and RIF (22/35) (Table 3). CLI and ERY were simultaneously inactive against 25 isolates: PRs 012, 017, 018, 027, 046 (Table 3). In 6 of these isolates, representing PRs 012 (2/2), 017 (2/3) and $046(2 / 2)$, both MLS $_{B}$ antibiotics showed MICs above $256 \mu \mathrm{g} / \mathrm{ml}$ [data not shown]. High resistance to MFX and RIF was noticed in PRs 027, 018 and 017, while PRs 012, 014, 046 and
115 were susceptible to both antibiotics (Table 3). LNZ-resistance was found in PR 017 (2/3) and PR $012(1 / 2)$. The respective strains were also resistant to CHL $(16->256 \mu \mathrm{g} / \mathrm{ml})$ (Table 3). IPM-resistance was noted in all PRs. Antibiotic GM MIC values resulted for the PRs are presented in Table 2.

In total, 12 resistance profiles, including 2-9 classes of antibiotics, were generated, as shown 


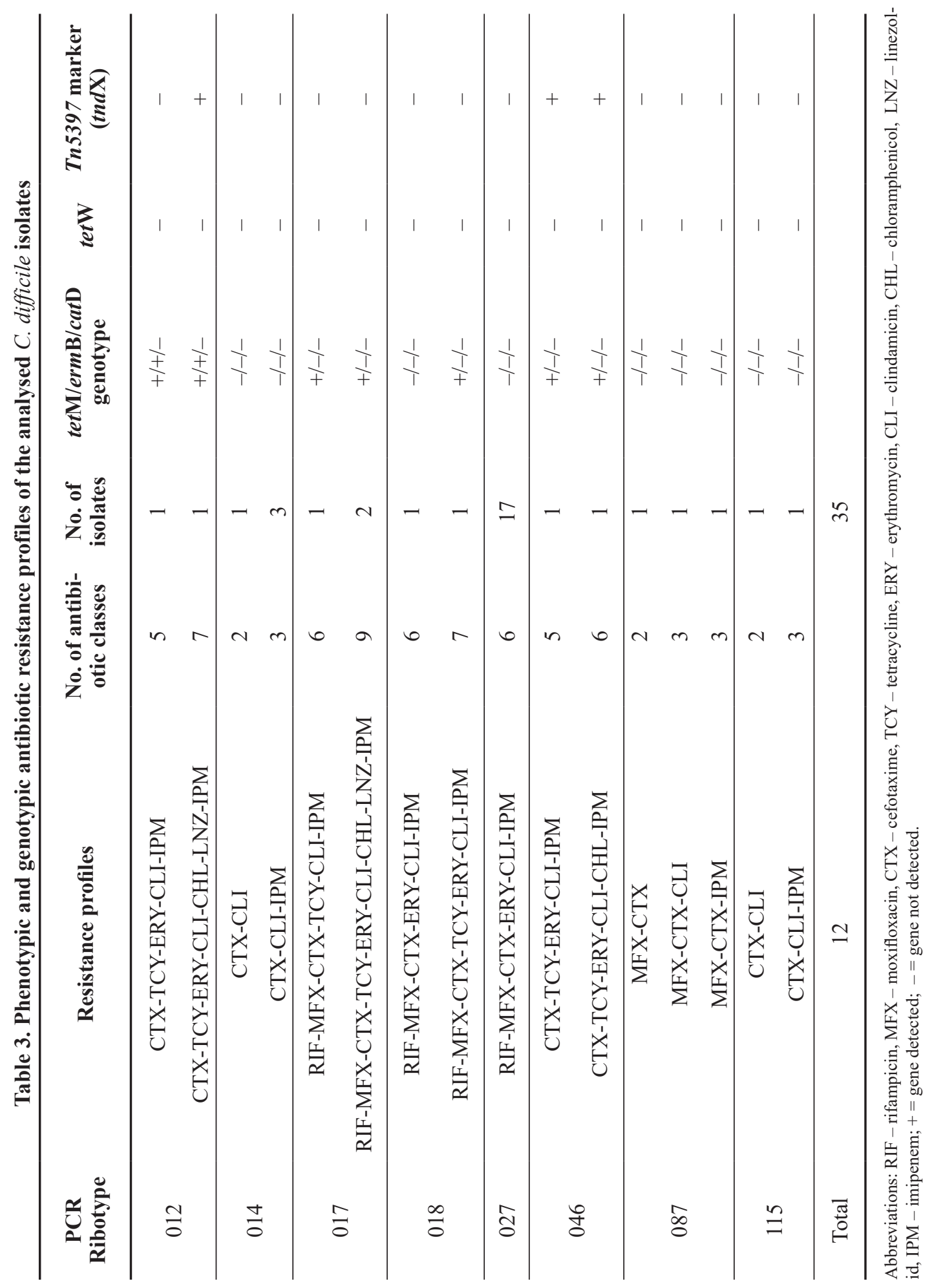


in Table 3. PR 027 isolates (17/17) showed a prevalent profile - RIF-MFX-CTX-ERY-CLIIPM (Figure 2). The LNZ-resistant isolates belonging to PR 017 expressed identical 9-antibiotic-resistance profiles (RIF-MFX-CTX-TCYERY-CLI-CHL-LNZ-IPM), while the third isolate showed a 6-antibiotic-profile (RIF-MFXCTX-TCY-CLI-IPM). Resistance to CTX, TCY, ERY, CLI and IPM coincided in PR 012 and PR 046 strains. Both PR 018 strains were non-susceptible to RIF, MFX, CTX, ERY, CLI and IPM. One also showed TCY-resistance. PR 087 isolates (3/3) were resistant to MFX and CTX, but were RIF-susceptible, although in other PRs tested, MFX- and RIF-resistance were associated. Two of them also expressed resistance to a third antibiotic - CLI or IPM (Table 3 ). PRs $014(4 / 4)$ and $115(2 / 2)$ were resistant to CTX and CLI. Three representatives of PR 014 and one PR 115 strain were also resistant to IPM (Table 3).

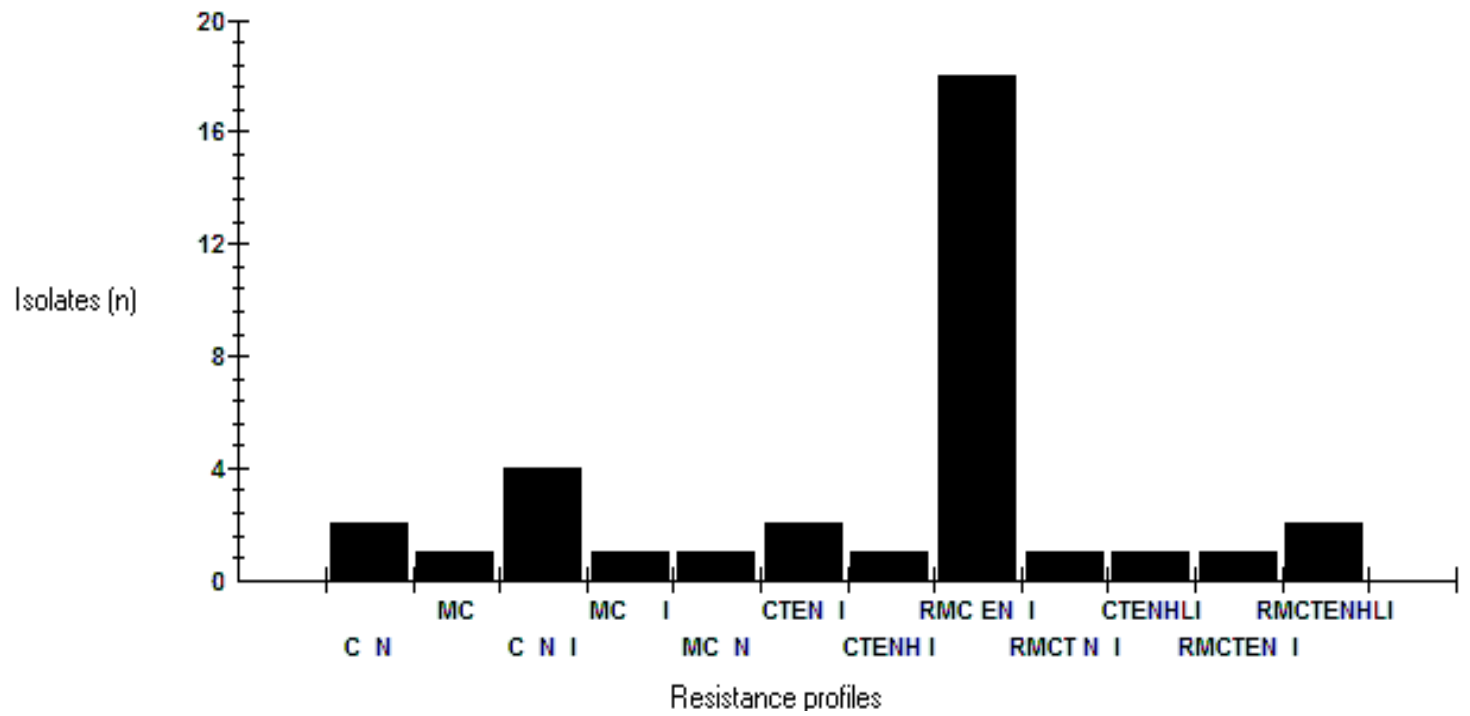

Figure 2. Distribution of antibiotic resistance profiles resulted for the studied group of isolates: CN - CTXCLI; MC - MFX-CTX; CNI - CTX-CLI-IPM; MCI - MFX-CTX-IPM; MCN - MFX-CTX-CLI; CTENI - CTX-TCY-ERY-CLI-IPM; CTENHI - CTX-TCY-ERY-CLI-CHL-IPM; RMCENI - RIF-MFX-CTXERY-CLI-IPM; RMCTNI - RIF-MFX-CTX-TCY-CLI-IPM; CTENHLI - CTX-TCY-ERY-CLI-CHLLNZ-IPM; RMCTENI - RIF-MFX-CTX-TCY-ERY-CLI-IPM; RMCTENHLI - RIF-MFX-CTX-TCYERY-CLI-CHL-LNZ-IPM.

\section{Molecular test results}

PCR results showed presence of TCY-resistance gene tet $\mathrm{M}$ in all the 8 phenotipically resistant isolates. Three of these strains, belonging to PR 046 (2/2) and PR 012 (1/2), were also positive for gene $t$ thd , specific toTn5397-like transposons [20]. None of the analysed isolates showed presence of the tet $\mathrm{W}$ gene. Both tet $\mathrm{M}$ and erm $\mathrm{B}$ were detected in two isolates belonging to PR 012, phenotypically resistant to TCY and $\mathrm{MLS}_{\mathrm{B}}$ (Table 3). The rest of the $\mathrm{MLS}_{\mathrm{B}}$-resistant isolates were ermB-negative. None of the CHL-resistant strains, belonging to PRs 012 (1/2), 017 (2/3) and $046(1 / 2)$, were positive for cat $\mathrm{D}$, suggesting that other molecular mechanisms are generating resistance (Table 3). Phenotypic TCY-, $\mathrm{MLS}_{\mathrm{B}}{ }^{-}$, or CHL-susceptible isolates did not show presence of genetic resistance determinants investigated in the study. Only three isolates showed phenotypic and genotypic concordance regarding susceptibility to TCY, MLS $_{\mathrm{B}}$ and CHL (Table 3). 


\section{Discussion}

In the context of insufficient antibiotic susceptibility data available at this moment to describe Romanian C. difficile strains causing CDI, the present study was focused on characterizing the susceptibility patterns of toxigenic isolates belonging to some of the PRs identified in Romanian CDI outbreaks or in sporadical cases: 012, 014, 017, 018, 027, 046, 087 and 115 [25, 28].

In vitro antibiotic susceptibility test results showed the studied group to be highly susceptible to VAN, MTR, TGC and TEC. Some of the PR 027 strains showed higher MIC values of MTR compared to other PRs, although the respective strains were classified as susceptible $[29,30]$ and the GM MIC $(0.137 \mu \mathrm{g} / \mathrm{ml})$ suggests high overall susceptibility levels. However, the method used in the study to determine antibiotic susceptibility of $C$. difficile is considered to have lower accuracy in detecting reduced susceptibility to MTR, compared to agar dilution based methods [33]. Recent reports show European PR 027 strains to have elevated MTR MICs $[12,15]$. Therefore reduced MTR-susceptibility could be present among Romanian strains as well. The analysed PR 027 strains were highly susceptible to VAN (GM MIC $=0.361 \mu \mathrm{g} / \mathrm{ml})$. High levels of in vitro activity of MTR and VAN against the PR 027 isolates do not guarantee the successful outcome of the antibiotic treatment of infections generated by these strains. PR 027 is known to have high recurrence potential [34]. Previous reports [33] suggested that the lack of correspondence between in vitro and in vivo antibiotic activity may be explained by pharmacokinetic properties for MTR - high upper gastrointestinal absorption leading to low concentration in the intestine [35] - or physiological characteristics ensuring survival of the strains. Recent in vivo studies demonstrated the competitive advantage of PR 027 over other ribotypes, the authors suggesting an increased potential of PR 027 strains in using limited environmental resources [36]. Re-colonization of the patient through contact with spore-contaminated surfaces could be another cause of relapse.

A high level of antimicrobial resistance, with heterogeneous resistance profiles, was characteristic for the studied group. A predominant profile - RIF-MFX-CTX-ERY-CLI-IPM - was, however, noticed. It was characteristic to PR 027 (17/17), but one PR 018 strain also expressed this pattern. Strains belonging to PRs 012, 017, 018, 027 and 046, previously described as MDR $[10,13]$, showed wide antibiotic resistance, especially PR 017 , resistant to antibiotics belonging to 9 classes.

MIC results of CTX, a third-generation cephalosporin $(64->256 \mu \mathrm{g} / \mathrm{ml})$, are in accordance with other reports in suggesting that the activity level of different cephalosporins may vary between individual strains, although this class of antibiotics is considered constitutively inactive against $C$. difficile [10]. The activity of other antibiotics - RIF, MFX, TCY, ERY, CLI, CHL, LNZ and IPM - was heterogeneous within the group, with both susceptible and non-susceptible strains. In most isolates, a previously reported phenotypic association between the activities of RIF and fourth-generation fluoroquinolone MFX [13] was noticed: PRs 017, 018 and 027 were highly resistant, while PRs 012, 046 and 115 were susceptible to both antibiotics. Exceptionally, isolates belonging to PR 087 were susceptible to RIF and resistant to MFX. Overall, high RIF- and MFX-resistance rates were characteristic for isolates belonging to different PRs. This finding represents a starting point for further analysis, in order to determine molecular patterns of rifampin- and fluoroquinolone-resistance in Romanian $C$. difficile isolates.

Moderate resistance rates were observed for TCY and CHL. MLS B $_{\mathrm{B}}$ antibiotics were predominantly inactive. All ribotypes were resistant to CLI. Among the CLI-resistant strains, 25 were 
also highly resistant to ERY (PRs 012, 017, 018, 027 and 046), coinciding with previous European study results $[12,15]$. Particularly, PR 027 was mostly associated with high ERY-resistance (GM MIC > $256 \mu \mathrm{g} / \mathrm{ml}$ ) and moderate CLI-resistance $(\mathrm{GM} \mathrm{MIC}=6.14 \mu \mathrm{g} / \mathrm{ml})$, while many European reports described PR 027 strains as highly resistant to CLI $[12,15]$. LNZ-resistance, previously reported in studies performed in Europe $[12,31]$, was detected here in strains belonging to PR 017 and PR 012, in association with high TCY, MLS $_{\mathrm{B}^{-}}$and CHL-resistance. The resistance noticed against IPM (GM MIC = $25.072 \mu \mathrm{g} / \mathrm{ml}$ ) could be reflecting the tendency to frequently prescribe carbapenem antibiotics in the country. Similar results, showing high levels of resistance in different PRs, were previously reported in Europe [15, 37].

Molecular test results suggest TCY-resistance to be mediated by tet $\mathrm{M}$ in the analysed $C$. difficile strains. The gene tet $\mathrm{W}$, reported in other studies as a co-determinant [22, 23], was not detected. Nevertheless, the possibility of other genetic mechanisms contributing to tetracycline-resistance in the analysed group is not to be ruled out. The presence of Tn5397-like specific gene $t n d \mathrm{X}$ in three of the tet $\mathrm{M}$-positive strains belonging to PRs 012 and 046, suggests that TCY-resistance was delivered through such an element, commonly transmitted between species of bacterial pathogens including $C$. difficile [21]. Only two strains (PR 012) showed presence of both tet $\mathrm{M}$ and erm $\mathrm{B}$ genes, although all TCY-resistant strains were also phenotypically resistant to $\mathrm{MLS}_{\mathrm{B}}$. The co-presence of tet $\mathrm{M}$ and erm $\mathrm{B}$ and the absence of tnd $\mathrm{X}$ in one of the two strains suggest the possibility of a Tn916-like element carrying both resistance genes to be integrated [38]. For the rest of the isolates, $\mathrm{MLS}_{\mathrm{B}}$-resistance could not be explained by ermB-encoded ribosomal metilation. In the last years, ermB-negative $C$. difficile strains phenotypically resistant to $\mathrm{MLS}_{\mathrm{B}}$, including PR 027 strains, have been described $[10,13]$. None of the analysed CHL-resistant isolates showed evidence of catD-mediated resistance, requiring further investigation of the molecular mechanisms of resistance. Heterogeneity of phenotypic and genotypic susceptibility to TCY, MLS $\mathrm{B}_{\mathrm{B}}$ and CHL was noticed in different strains belonging to the same ribotype (e.g. PRs 017, 046, 087), suggesting that antibiotic resistance is acquired through horizontal transmission of genetic determinants between individual strains and is not necessarily ribotype-dependent. LNZ-resistance, previously reported in studies performed in Europe [12, 31], was detected in strains belonging to PR 017, in association with high $\mathrm{MLS}_{\mathrm{B}}-$ and CHL-resistance. However, the absence of genes ermB and cat $\mathrm{D}$ in these strains allows speculating that other molecular determinants, such as MDR genes, could be inducing resistance [31, 39].

In conclusion, the clinical isolates analysed in this study have high levels of antimicrobial resistance, especially to wide-spectrum antibiotics representing cephalosporins, $\mathrm{MLS}_{\mathrm{B}}$, rifampins, fluoroquinolones and carbapenems, considered risk factors for development of $C$. difficile infection, along with advanced age and hospitalization $[1,8,10,40]$. Multi-drug resistance may be attributed to the majority of isolates, resistant to at least five classes of antibiotics [13]. Tetracycline-resistance was attributed to the tet $\mathrm{M}$ gene. As most $\mathrm{MLS}_{\mathrm{B}}$-resistant isolates were ermB-negative and no chloramphenicol-resistant isolates showed presence of cat $\mathrm{D}$, molecular resistance mechanisms against these antibiotics remain unclear and are to be analysed more thoroughly in future studies. The findings in the present study support the assertion that extensive administration of rifampin, carbapenems such as imipenem, fluoroquinolones, cephalosporins or $\mathrm{MLS}_{\mathrm{B}}$ $[6,15,21,40,41]$, could involve high risk of CDI development and emergence of outbreaks, especially with epidemic PRs 027 or 017 . In contrast, taking into consideration antibiotics 
demonstrated to be highly active against $C$. difficile, when selecting treatment for various types of infections, could contribute to minimising the risk of CDI spread.

This study underlines, once again, the impact of over prescription of wide-spectrum antibiotics on transmission of antibiotic resistance and emergence of highly epidemic $C$. difficile strains generating outbreaks. In our country, reckless administration of antibiotics has been an issue for many years, contributing to high incidence of infections caused by $C$. difficile in hospital settings or in the community and also making CDI prevention difficult.

\section{Acknowledgements}

This study was partially funded by the National Authority for Scientific Research and Innovation (NASR) of Romanian Ministry of Research and Innovation, through Project "Nucleu" PN09220104. I thank Dr. Marina Pana for her support in preparing the manuscript.

\section{List of abbreviations}

PMC - pseudomembranous colitis;

CDI - C. difficile infection;

TcdA - toxin C. difficile A (enterotoxin);

TcdB - toxin C. difficile B (cytotoxin);

$\mathrm{CDT}-C$. difficile toxin (binary toxin);

$\mathrm{PR}$ - PCR ribotype;

MDR - multi-drug resistant;

$\mathrm{MLS}_{\mathrm{B}}$ - Macrolide-Lincosamide-Streptogramin

$\mathrm{B}$;

erm B - class B erythromycin ribosomal methylase gene;

$\mathrm{RPP}$ - ribosome protection protein;

tet $\mathrm{M}$ - tetracycline resistance protein class $\mathrm{M}$ (TetM) gene;

tet $\mathrm{W}$ - tetracycline resistance protein class $\mathrm{W}$ (TetW) gene;

tnd $\mathrm{X}$ - conjugative transposon site-specific recombinase (TndX) gene; cat $\mathrm{D}$ - chloramphenicol acetyltransferase

(CatD) gene;

VAN - vancomycin;

MTR - metronidazole;

TGC - tigecycline;

TEC - teicoplanin;

RIF - rifampicin;

MFX - moxifloxacin;

CTX - cefotaxime;

TCY - tetracycline;

ERY - erythromycin;

CLI - clindamycin;

CHL - chloramphenicol;

LNZ - linezolid;

IPM - imipenem;

ECOFF - epidemiological cut-off;

$\mathrm{S}$ - susceptible;

I - intermediary;

$\mathrm{R}$ - resistant;

$\mathrm{CN}$ - CTX-CLI;

MC - MFX-CTX;

CNI - CTX-CLI-IPM;

MCI - MFX-CTX-IPM;

MCN - MFX-CTX-CLI;

CTENI - CTX-TCY-ERY-CLI-IPM;

CTENHI - CTX-TCY-ERY-CLI-CHL-IPM;

RMCENI - RIF-MFX-CTX-ERY-CLI-IPM;

CTENHLI - CTX-TCY-ERY-CLI-CHL-LNZ-

IPM;

RMCTNI - RIF-MFX-CTX-TCY-CLI-IPM;

RMCTENI - RIF-MFX-CTX-TCY-ERY-CLI-

IPM;

RMCTENHLI - RIF-MFX-CTX-TCY-ERYCLI-CHL-LNZ-IPM.

\section{References}

1. Bartlett JG, Gerding DN. Clinical recognition and diagnosis of Clostridium difficile infection. Clin Infect Dis. 2008 Jan;46(Suppl 1):S12-S18. DOI: 10.1086/521863

2. Bloomfield LE, Riley TV. Epidemiology and risk factors for community-associated Clostridium difficile infection: a narrative review. Infect Dis Ther. 2016 Sep;5(3):231-51. DOI: 10.1007/s40121-016-0117-y 
3. Rupnik M. Is Clostridium difficile-associated infection a potentially zoonotic and foodborne disease? Clin Microbiol Infect. 2007 May;13(5):457-9. DOI: 10.1111/j.1469-0691.2007.01687.x

4. Hall IC, O'Toole E. Intestinal flora in new-born infants with a description of a new pathogenic anaerobe, Bacillus difficilis. Am J Dis Child. 1935;49(2):390-402. DOI: 10.1001/archpedi.1935.01970020105010

5. Voth DE, Ballard JD. Clostridium difficile toxins: mechanism of action and role in disease. Clin Microbiol Rev. 2005 Apr;18(2):247-263. DOI: 10.1128/ CMR.18.2.247-263.2005

6. Wilcox MH, Chalmers JD, Nord CE, Freeman J, Bouza E. Role of cephalosporins in the era of Clostridium difficile infection. J Antimicrob Chemother. 2017 Jan;72(1):1-18. DOI: 10.1093/jac/dkw385

7. Bartlett JG, Onderdonk AB, Cisneros RL, Kasper DL. Clindamycin-associated colitis due to a toxin-producing species of Clostridium in hamsters. J Infect Dis. 1977 Nov;136(5):701-5. DOI: 10.1093/infdis/136.5.701

8. Owens Jr RC, Donskey CJ, Gaynes RP, Loo VG, Muto CA. Antimicrobial-associated risk factors for Clostridium difficile infection. Clin Infect Dis. 2008 Jan;46(Suppl 1):S19-S31. DOI: 10.1086/521859

9. Baines SD, Wilcox MH. Antimicrobial resistance and reduced susceptibility in Clostridium difficile: potential consequences for induction, treatment, and recurrence of C. difficile infection. Antibiotics (Basel). 2015 Sep;4(3):267-8. DOI: 10.3390/antibiotics4030267

10. Spigaglia P. Recent advances in the understanding of antibiotic resistance in Clostridium difficile infection. Ther Adv Infect Dis. 2016 Feb;3(1):23-42. DOI: $10.1177 / 2049936115622891$

11. McFarland LV, Ozen M, Dinleyici EC, Goh S. Comparison of pediatric and adult antibiotic-associated diarrhea and Clostridium difficile infections. World J Gastroenterol. 2016 Mar;22(11):3078-104. DOI: 10.3748/ wjg.v22.i11.3078

12. Freeman J, Vernon J, Vickers R, Wilcox MH. Susceptibility of Clostridium difficile isolates of varying antimicrobial resistance phenotypes to SMT19969 and 11 comparators. Antimicrob Agents Chemother. 2016 Jan;60(1):689-692. DOI: 10.1128/AAC.02000-15

13. Spigaglia P, Barbanti F, Mastrantonio P, on behalf of the European Study Group on Clostridium difficile (ESGCD). Multidrug resistance in European Clostridium difficile clinical isolates. J Antimicrob Chemother. 2011 Oct;66(10):2227-34. DOI: 10.1093/jac/dkr292

14. Vardakas KZ, Konstantelias AA, Loizidis G, Rafailidis PI, Falagas ME. Risk factors for development of Clostridium difficile infection due to $\mathrm{BI} /$ NAP1/027 strain: a meta-analysis. Int J Infect Dis. 2012 Nov;16(11):e768-e773/. DOI: 10.1016/j. ijid.2012.07.010

15. Freeman J, Vernon J, Morris K, Nicholson S, Todhunt- er S, Longshaw C, et al. Pan-European longitudinal surveillance of antibiotic resistance among prevalent Clostridium difficile ribotypes. Clin Microbiol Infect. 2015 Mar; 21(3):248.e9-248.e16. DOI: 10.1016/j. cmi.2014.09.017

16. Farrow KA, Lyras D, Rood JI. Genomic analysis of the erythromycin resistance element Tn5398 from Clostridium difficile. Microbiology. 2001 Oct;147(10):271728. DOI: 10.1099/00221287-147-10-2717

17. Spigaglia P, Mastrantonio P. Comparative analysis of Clostridium difficile clinical isolates belonging to different genetic lineages and time periods. J Med Microbiol. 2004 Nov;53(11):1129-36. DOI: 10.1099/ jmm.0.45682-0

18. Dönhöfer A, Franckenberga S, Wicklesa S, Berninghausena O, Beckmann R, Wilson DN. Structural basis for TetM-mediated tetracycline resistance. Proc Natl Acad Sci U S A. 2012 Oct;109(42):16900-5. DOI: 10.1073/pnas. 1208037109

19. Mullany P, Wilks M, Lamb I, Clayton C, Wren B, Tabaqchali $\mathrm{S}$. Genetic analysis of a tetracycline resistance element from Clostridium difficile and its conjugal transfer to and from Bacillus subtilis. J Gen Microbiol. 1990 Jul;136(7):1343-9. DOI: 10.1099/00221287-1367-1343

20. Wang H, Mullany P. The large resolvase TndX is required and sufficient for integration and excision of derivatives of the novel conjugative transposon Tn5397. J Bacteriol. 2000 Dec;182(23):6577-83. DOI: 10.1128/ JB.182.23.6577-6583.2000

21. Spigaglia P, Carucci V, Barbanti F, Mastrantonio P. ErmB determinants and Tn916-like elements in clinical isolates of Clostridium difficile. Antimicrob Agents Chemother. 2005 Jun;49(6):2550-3. DOI: 10.1128/ AAC.49.6.2550-2553.2005

22. Fry PR, Thakur S, Abley M, Gebreyesa WA. Antimicrobial resistance, toxinotype, and genotypic profiling of Clostridium difficile isolates of swine origin. J Clin Microbiol. 2012 Jul;50(7):2366-72. DOI: 10.1128/ JCM.06581-11

23. Spigaglia P, Barbanti F, Mastrantonio P. Tetracycline resistance gene tet $(\mathrm{W})$ in the pathogenic bacterium Clostridium difficile. Antimicrob Agents Chemother. 2008 Feb;52(2):770-3. DOI: 10.1128/AAC.00957-07

24. Kuijper EJ, Coignard B, Tüll P, on behalf of the ESCMID Study Group for Clostridium difficile (ESGCD), EU Member States and the European Centre for Disease Prevention and Control (ECDC). Emergence of Clostridium difficile-associated disease in North America and Europe. Clin Microbiol Infect. 2006 Oct;12(Suppl. 6):2-18. DOI: 10.1111/j.1469-0691.2006.01580.x

25. Popescu GA, Șerban R, Pistol A, Niculcea A, Preda A, Lemeni D, et al. Clinical and microbiological characterization of Clostridium difficile infection in Romania (2013-2014); a hospital based study. BMC Infect Dis. 
2014a;14(Suppl 7):o24. DOI: 10.1186/1471-2334-14$\mathrm{S} 7-\mathrm{O} 24$

26. Popescu GA, Florea D, Rafila A. Clostridium difficile is emerging in Romania: a story of 027 ribotype and excessive antibiotic consumption. J Gastrointestin Liver Dis. 2014b;23(3):342-3.

27. Florea D, Huhulescu S, Indra A, Badicut I, Rafila A, Otelea D, et al. PCR coupled with mass-spectrometry for detection of Clostridium difficile virulence markers during the emergence of ribotype 027 in Bucharest area. Rev Romana Med Lab. 2015;23(4):449-55. DOI:10.1515/rrlm-2015-0044

28. Macovei IS, Lemeni D, Usein CR, Șerban R, Niculcea A, Popescu GA, et al. The use of PCR Ribotyping for molecular typing of clinically significant Clostridium difficile Romanian isolates. Rom Biotechnol Lett. 2017;22(5).

29. The European Committee on Antimicrobial Susceptibility Testing. Breakpoint tables for interpretation of MICs and zone diameters, version 7.1. 2017; http:// www.eucast.org/fileadmin/src/media/PDFs/EUCAST_ files/Breakpoint_tables/v_7.1_Breakpoint.

30. Clinical and Laboratory Standards Institute. Performance standards for antimicrobial susceptibility testing. CLSI document M100-27th ed. 2017:96-100.

31. Marin M, Martín A, Alcalá LM, Cercenado E, Iglesias C, Reigadas E, et al. Clostridium difficile isolates with high linezolid MICs harbor the multiresistance gene cfr. Antimicrob Agents Chemother. 2015 Jan;59(1):586-9. DOI: 10.1128/AAC.04082-14

32. Marchese A, Ramirez M, Schito GC, Tomasz A. Molecular epidemiology of penicillin-resistant Streptococcus pneumoniae isolates recovered in Italy from 1993 to 1996. J Clin Microbiol. 1998 Oct;36(10):2944-9.

33. Baines SD, O'Connor R, Freeman J, Fawley WN, Harmanus C, Mastrantonio P, et al. Emergence of reduced susceptibility to metronidazole in Clostridium difficile. J Antimicrob Chemother. 2008 Nov;62(5):1046-52. DOI: $10.1093 / \mathrm{jac} / \mathrm{dkn} 313$

34. Marsh JW, Arora R, Schlackman JL, Shutt KA, Curry SR, Harrison LH. Association of relapse of Clostridium difficile disease with BI/NAP1/027. J Clin Microbiol. 2012 Dec;50(12):4078-82. DOI: 10.1128/JCM.0229112

35. Cherian PT, Wu X, Yang L, Scarborough JS, Singh AP, Alam ZA, et al. Gastrointestinal localization of metronidazole by a lactobacilli-inspired tetramic acid motif improves treatment outcomes in the hamster model of Clostridium difficile infection. J Antimicrob Chemother. 2015 Nov;70(11):3061-9. DOI: 10.1093/jac/dkv231

36. Robinson CD, Auchtung JM, Collins J, Britton RA. Epidemic Clostridium difficile strains demonstrate increased competitive fitness compared to nonepidemic isolates. Infect Immun. $2014 \mathrm{Jul} ; 82(7): 2815-25$. DOI: 10.1128/IAI.01524-14

37. Lachowicz D, Pituch H, Obuch-Woszczatyński P. Antimicrobial susceptibility patterns of Clostridium difficile strains belonging to different polymerase chain reaction ribotypes isolated in Poland in 2012. Anaerobe. 2015 Feb;31:37-41. DOI: 10.1016/j.anaerobe.2014.09.004

38. Spigaglia P, Barbanti F, Mastrantonio P. Detection of a genetic linkage between genes coding for resistance to tetracycline and erythromycin in Clostridium difficile. Microb Drug Resist. 2007;13(2):90-5. DOI: 10.1089/ mdr.2007.723

39. Knight DR, Elliott B, Chang BJ, Perkins TT, Riley TV. Diversity and evolution in the genome of Clostridium difficile. Clin Microbiol Rev. 2015 Jul;28(3):721-41. DOI: 10.1128/CMR.00127-14

40. Büchler AC, Rampini SK, Stelling S, Ledergerber B, Peter S, Schweiger A, et al. Antibiotic susceptibility of Clostridium difficile is similar worldwide over two decades despite widespread use of broad-spectrum antibiotics: an analysis done at the University Hospital of $\mathrm{Zu}-$ rich. BMC Infect Dis. 2014 Nov; 14:607. DOI: 10.1186/ s12879-014-0607-z

41. Curry SR, Marsh JW, Shutt KA, Muto CA, O'Leary MM, Saul MI, et al. High frequency of rifampin resistance identified in an epidemic Clostridium difficile clone from a large teaching hospital. Clin Infect Dis. 2009 Feb;48(4):425-9. DOI: 10.1086/596315 